\title{
Natural Resources Research: Acknowledgement of Reviewers for 2020
}

\author{
John Carranza, Editor-in-Chief, Natural Resources Research ${ }^{1,2}$
}

Accepted 21 January 2021
Published online: 1 March 2021

Improving and maintaining the standard of papers in this journal depend on the selfless time and effort of the many scientists who review manuscripts. We acknowledge and thank the following individuals for reviewing manuscripts submitted to our journal during 2020; some of them have reviewed more than one manuscript.

\author{
Alpana \\ Hazem Abd EL Hamid \\ Mohamed Abdel-Fattah \\ Mohammad Abdideh \\ A.M. Abdulaziz \\ Maysam Abedi \\ M.J. Abrams \\ Frits Agterberg \\ Arif Ahamad \\ Mohammed Ahmed \\ W. Ahmed \\ Mehmet Aksoy \\ K. Al Amari \\ Alaa Al-Abadi \\ I.M. Al-Alfy \\ Meysam Alizamir \\ José António Almeida \\ Watheq Al-Mudhafar \\ Hasan Al-Saedi \\ A.A. Al-Shuhail \\ Sergey Alyaev \\ Hakimeh Amanipoor \\ Alexey Amdur \\ Tiri Ammar \\ W. Ampomah
}

P.Ø. Andersen

F.H. Andriamasinoro

A. Ansari

H.R. Ansari

Evangelina Apparicio

R. Archer

Zakari Arétouyap

Danial Jahed Armaghani

Margaret Armstrong

Omid Asghari

Ahmad Ashtari

Mehdi Assareh

Emil Attanasi

Özgür Avsar

M. Awais

L. Azevedo

Y. Azimi

Jing Ba

A. Baghban

M. Bagheri

Gang Bai

Yuhu Bai

Zujin Bai

Elham Bakhshi

Ezzeddin Bakhtavar
Sally Barritt

Georgios Bartzas

Rahim Barzegar

Biraj Basak

I.O. Bayuk

Daniel Beaufort

Belew Bekele

Jörg Benndorf

A. Bernava

Kathryn Bethune

P.K. Bikkina

Arkoprovo Biswas

S.H. Bloomberg

Nikolai Bobylev

Jeff Boisvert

Graeme Bonham-Carter

R. Bowell

Antonella Buccianti

M. Bucha

X.N. Bui

A.K. Burnham

Natalie Caciagli

Jihua Cai

Eray Can

Han Cao

${ }^{1}$ Durban, South Africa.

${ }^{2}$ To whom correspondence should be addressed; e-mail: ejmcarranza@gmail.com 
W. Cao

Silvia Castellaro

Liliana Castro

Liping Chang

X. Chang

Rima Chatterjee

Snehamoy Chatterjee

Bin Chen

Jui-Sheng Chen

Liang Chen

Peiyuan Chen

Tianyu Chen

Wei Chen

Yanni Chen

Yuhang Chen

Guoxiong Chen

Yongliang Chen

Jianwei Cheng

A.G. Chiwona

Tingxiang Chu

Giuseppe Cianflone

Giuseppe Cillis

Timothy Coburn

D. Cohen

Resul Cömert

Juan Contreras

Joshua Coyan

Luz Adriana Cuartas

A.C. da Costa Martins

Hooman Dadashzadeh Ahari

Yasin Dagasan

Shifeng Dai

A. Das

Jew Das

Sumit Das

L. Dascalescu

Sumangal Dasgupta

Y. Daud

Rhys S. Davies

Davoud Davoudi

Moghaddam

Eimear Deady

M. Dejam

M. Demİrci

J. Deng

Clayton Deutsch

A. Dhar

Zein Diab

X. Ding

M.A.P. Dinis

B.M. Dodamani

B.M. Dodamani
Shuheng Du

Semih Ekercin

Georges Ekosse

Mahmoud Elhussein

Salaheldin Elkatatny

S.I. Elmahdy

Matthew Elmes

Ayman El-Midany

A.M.A. El-Sayed

Xavier Emery

Sk. Md. Equeenuddin

M.O. Eremin

S. Esmaeili

B. Esrafili-Dizaji

Khalid Essa

Hatem Ewida

Chaojun Fan

G. Fan

Y. Fan

Bojiang fan

S. Farzin

M. Fatehi

S.M. Fatemi

Leonardo Feltrin

Guorui Feng

Arianne Ford

Andrea Förster

F. Fouedjio

Hakim Gabtni

V. Gangrade

Shib Ganguli

Yukun Gao

Yue Gaowei

Michael Gazley

Zhaolong Ge

KHaled Gemail

Bekir Genc

Nyakno J. George

Dimitrios Gerogiorgis

Ali Ghabeishavi

G. Ghiglieri

H. Ghorbani

Omid Ghorbanzadeh

Mohamed Gobashy

Modreck Gomo

Mario Goncalves

Qingjie Gong

H. Govil

D.D. Gregory

Eric Grunsky

Qingyu Guan

R. Guellala
A.G. Gunn

A. Gunning

Chen Guo

Hongyu Guo

S. Guo

Tiankui Guo

Wenmu Guo

Yafei Guo

Sergey Gutnikov

Mohsen Hajihassani

N. Hamdani

D. Han

Jeff R. Harris

M.K. Hasan

Mahdi Hasanipanah

Bodhisatwa Hazra

$\mathrm{Li} \mathrm{He}$

Mohammad Heidary

Ahmed Henaish

Azim Heydari

R.E. Hincapie

Bizhan Honarvar

Shawn Hood

Mikael Höök

Mohammad Hosseini

S.M. Hosseini

Haihai Hou

James Hower

Karel Hron

Ping-Cheng Hsieh

B. Huang

Gang Huang

Tianming Huang

Xiaowen Huang

I.N. Hulea

Zhipeng Huo

Johnson Ibuot

Adonis Ichim

Rami Idan

M. Irani

Hiromasa Ishikawa

Abolfazl Jaafari

Bahram Jafrasteh

Ashkan Jahanbani Ghahfarokhi

Mohsen Jalali

Chokri Jallouli

J.A. Jarzyna

Jerry Jensen

Hancheng Ji

Fujie Jiang

Yi Jin 


Linhai Jing
Behshad Jodeiri Shokri
Birgir Johannesson
Yang Ju
Dedi Juandi
A.M. Jubb
Mark Kaiser
Shams Kalam
Bahareh Kalantar
Azim Kalantariasl
Y. Kang
Abdulkadir Karadogan
Hamid Kardan Moghaddam
Vahid Karimi
G.P. Karmakar
Ram Chandar Karra
Milton Kataka
Jarrod Kath
Masaji Kato
Witold Kawalec
O.I. Kazanin
Tirumalesh Keesari
Akos Kereszturi
M.R. Khaleghi
Perveiz Khalid
Mohamed Khalifa
Faisal Khan
Junaid Khan
S.Z. Khan
Manoj Khandelwal
E. Khodapanah
Hyunna Kim
Barry King
N. Knox
Mohammadreza
$\quad$ Koopialipoor
Vanessa Koppe
Oliver Kreuzer
L.T. Krupskaya
Jerry Kuma
Harinandan Kumar
P.C. Kumar
Mustafa Kumral
Marcus Kunzmann
Ilgin Kursun
Agustín Laguarda
Jin Lai
E. Laloy
K.B. Larionov
K. Lawal
S.M. Lawan
Jin-Yong Lee

S. Leinß
Biao Li
Bo Li
Chengwu Li
Hu Li
Lei Li
Liyun Li
M. Li
Qingmiao Li
S. Li
Xuelong Li
Yang Li
Yong Li
Yuwei Li
Bobo Li
Yong LI
Shun Liang
K.P. Lin
Ang Liu
Guangfeng Liu
Huan Liu
Jingshou Liu
K. Liu
Lei Liu
Wei Liu
X. Liu
Xianfeng Liu
Yaoru Liu
Yue Liu
Zhiheng Liu
Tong Liu
Yanbao Liu
Chunyan Lu
Haifeng Lu
Y. Lu
F.A. Lucay
Xiaorong Luo
Jing Ma
L. Ma
Yankun Ma
Mohammad Maanijou
Nasser Madani
H. Mahani
Pankajini Mahanta
Ahmed Abdulhamid
P. Mahzari
Sudip Maity
Bahram Malekmohammadi
A.A. Malinowska
A. Mandal
David Manning

B. Manoj

A.K. Manshad Ahmed Mansour Henryk Marszałek R. Masoudi Micòl Mastrocicco Mitsuo Matsumoto J. McKinley A. Mehmani Hamzeh Mehrabi Gang Mei A. Meilijson Shangzhi Meng Zhaoping Meng Stathis Michaelides Ionut Minea Richard Minnitt Toru Mogi M.A. Mohammadi Ibrahim Mohialdeen Thomas Monecke Masoud Monjezi Jacek Mucha Ute Mueller Munyaradzi Mujuru B. Mukherjee T. Murphy Bassem Nabawy D.R. Nagaraj S.A. Naghibi S. Nandy Hoang Nguyen Baisheng Nie Hima Nikafshan Rad M.E. Niri Ryan Noble Younes Noorollahi I. Nowrouzi Glen Nwaila Seokhoon Oh G.P. Oliveira M. Omeje Adeyinka Omotehinse Julian Ortiz M. Osanloo Boris Osovetsky John P. O'Sullivan Z. Ouyang C.A. Oyelami Kehinde Oyeyemi A. Pajdak D. Palmer 
Guocheng Pan

Rongkun Pan

Zhejun Pan

M. Panahi

Eulogio Pardo

R. Parsaei

Greg Partington

Abhishek Pathak

Shray Pathak

Alberto Patiño Douce

Fanglle Peng

Wenfu Peng

Xiaolong Peng

L. Pérez-Barnuevo

B.T. Pham

Leonardo Piccinini

H.R. Pourghasemi

Y. Pourrahimian

Christophe Preux

C. Pritzel

Rosa M. Prol-Ledesma

Ó. Pueyo Anchuela

Michael Pyrcz

Minfang Qi

Rui Qiao

Li Qingmiao

Liming Qiu

Zou Quanle

G.R. Quezada

Ahmed Radwan

M.S. Rahman

Omid Rahmati

S.N. Rai

C. Rainieri

S. Rajendran

Otmane Raji

H. Ranjbar

T.M. Rasmussen

L. Rddad

Zongping Ren

D. Renard

Ali Rezaei

M. Rezaie-Balf

J. Ribeiro

T. L. Rong

Edoardo Rossi

D. Rousseau

Marcello Ruberti

Zhenhua Rui

S. Sreekesh

S. Sadeghfam

Behnam Sadeghi
P.K. Sahoo

Satiprasad Sahoo

Saif Said

Akira Saito

Emmanuel Sakala

M. Salari

Alireza Salimi

Abani R. Samal

Biswajit Samanta

Oluseun Sanuade

Bhabesh Sarkar

K.N. Sayl

D.R. Schmitt

Jacopo Seccatore

Akram Seifi

Alexander Selemo

Souvik Sen

Belkhir Settou

A. Shabani

Imran Khan Shaik

M.R. Shalaby

Chang'an Shan

Delei Shang

Zhenlu Shao

T. Sharma

Jiping She

Jian Shen

Xianda Shen

Sergei Shevyrev

Peng Shi

Qingmin Shi

S. Shi

Wenjiao Shi

Xiuzhi Shi

Amin Shokrollahi

C. Shu

L. Shu

Qin Shuai

N.A. Siddiqui

Parveen Sihag

Luis Silva

Donald Singer

Ajay Singh

Gurjeet Singh

Omvir Singh

Kunwar Singh

Mike Sips

Anirbid Sircar

Mehrdad Soleimani

Monfared

Dazhao Song

Hongqing Song
Zeyang Song

Vinoth Srinivasan

I.R. Stakhovsky

S. Stojadinović

Yuantian Sun

B. Sun

Chengzhen Sun

Jianmeng Sun

Pingchang Sun

Tao Sun

Zheng Sun

Zhuang Sun

A. Surendar

O. Sylvester

A. Szafranek-Nakonieczna

Mehdi Tadayoni

K. Tadyszak

Pejman Tahmasebi

Faham Tahmasebinia

Eduardo Takafuji

Ryohei Takahashi

O. Talavera

Hassan Talebi

B. Tan

Jizhou Tang

M.H. Tangestani

Shu Tao

Evdokia Tapoglou

Boleslav Taraba

Afshin Tatar

Olivier Tavchandjian

Erhan Tercan

J.M. Thabit

Mainak Thakur

Christien Thiart

Weichao Tian

Prarabdh Tiwari

Farshid Torabi

Japan Trivedi

P. Tsangaratos

Shihao Tu

R.N. Turukmane

S. Tuylu

Nicholas Utting

Behzad Vaferi

Walter Valery

Bradley Van Gosen

J.A. Vargas-Guzmán

P.D. Vermeulen

Mauro Viccaro

M.G.C. Vincentelli

H.J. Vinegar 
S. Viti

Alaaddin Vural

Nicola Wagner

Chaolin Wang

Anjian Wang

Boyang Wang

Changxiang Wang

Daobing Wang

Dengke Wang

Dongying Wang

Gang Wang

Gongwen Wang

Guozhi Wang

Haiyan Wang

Hongyan Wang

Jian Wang

Jiang-Hai Wang

Jintang Wang

Kai Wang

Qian Wang

R.Z. Wang

Shaofeng Wang

Shen Wang

Wenhui Wang

Xiaoqi Wang

Yongliang Wang

Zhaofeng Wang

Jianping Wei

J. Weiss

Ron Wong

David Wood

Caifang Wu

Kunyu Wu

Zhaodong Xi

Tongqiang Xia

C. Xian
Dong Xiao

Yong Xiao

Jianyong Xie

Shuyun Xie

Wei Xie

$\mathrm{Ke} \mathrm{Xu}$

Jorge K. Yamamoto

Chunhua Yan

Hao Yan

Dazhi Yang

Dong Yang

Feng Yang

Hongying Yang

Leilei Yang

Rui Yang

Xianyu Yang

Yongkang Yang

Y. Yao

Zaher Mundher Yaseen

Qamar Yasin

Mahmut Yavuz

Qing Ye

Graham Yielding

Dawei Yin

Yanshu Yin

Mahyar Yousefi

Reza Yousefzadeh

Bo $\mathrm{Yu}$

Wei Yu

Yang $\mathrm{Yu}$

Feng Yuan

R.S. Yuan

Sanyi Yuan

Tianxiang Yue

Jie Zang

Sohrab Zendehboudi
Beibei Zhang

Daojun Zhang

Dongming Zhang

Junfei Zhang

Lei Zhang

Liang Zhang

Pengyan Zhang

Songhang Zhang

Xianlong Zhang

Xianmin Zhang

Xiaoyang Zhang

Yihuai Zhang

Yongjiang Zhang

Zetian Zhang

Zhengguang Zhang

Zhenzihao Zhang

Dong Zhao

Yangsheng Zhao

Zhiqiang Zhao

P. Zheng

T. Zheng

Yijun Zheng

Ruizhi Zhong

Fengde Zhou

Jian Zhou

Wenzuo Zhou

Xiaoping Zhou

Yinbo Zhou

L. Zhu

Wenbin Zhu

Yongguang Zhu

Mingjun Zou

Lihua Zuo

Shudi Zuo

Jianping Zuo

We apologize for any inadvertent errors or omissions. 